\title{
A CHARACTERIZATION OF FINITELY JUNCTIONED CONTINUA
}

\author{
EDWIN DUDA AND PAWEL KRUPSKI
}

(Communicated by James E. West)

\begin{abstract}
Metric continua which are $k$-junctioned are defined and characterized. Furthermore such continua are shown to be invariant under open or monotone mappings.
\end{abstract}

A metric continuum is said to be $k$-junctioned if it is an inverse limit of graphs each of which has at most $k$ branch points, with surjective bonding maps. A continuum is called finitely junctioned if it is $k$-junctioned for some integer $k \geq 0$.

Finitely junctioned continua were considered in relation to homogeneity in $[2,4,7]$.

It follows from the definition that a continuum is 0 -junctioned if and only if it is either chainable or circularly chainable. In this note we obtain an analogous characterization for all finitely junctioned continua (Theorem 1). It follows, for example, that if each proper subcontinuum of a nondegenerate metric continuum is chainable, then the continuum is 1 -junctioned. The result is the best possible (see [5]).

Another interesting and easy consequence of Theorem 1 is the invariance of $k$-junctionedness under monotone and open mappings. It is known that a monotone image of a chainable continuum is chainable [1] and a monotone image of a circularly chainable continuum is circularly chainable [3]. We extend these results to $k$-junctioned continua (Theorem 2). An open image of a chainable continuum is chainable, by [8], but the old problem of whether an open image of a circularly chainable continuum has to be 0 -junctioned remains, in general, unsolved (positive answers are known for solenoids [6] or under the additional assumption that the range space is decomposable [3]). It follows from our Theorem 3 that an open image of a circularly chainable continuum is 1 -junctioned.

An element of a cover of a space is called a junction element if it meets at least three other elements of the cover. A collection of open subsets $U_{1}, U_{2}, \ldots, U_{n}$ of a space $X$ is a chain (circular chain) in $X$ if $U_{i} \cap U_{j} \neq \varnothing \Leftrightarrow|i-j| \leq$ $1(|i-j| \leq 1$ or $\{i, j\}=\{1, n\})$. We will use an equivalent definition of $k$ junctioned continua in terms of covers. A metric nondegenerate continuum $X$

Received by the editors January 4, 1991. This paper was presented at the 24th annual Spring Topology Conference held at Southwest Texas State University, San Marcos, Texas, April 5-7, 1990.

1991 Mathematics Subject Classification. Primary 54F20, 54C10; Secondary 54F15, 54F50. 
is $k$-junctioned if and only if for every $\varepsilon>0$ there exists a finite open $\varepsilon$-cover of $X$ of order one with at most $k$-junction elements. A metric continuum $X$ is chainable (circularly chainable) if for every $\varepsilon>0$ there exists an $\varepsilon$-chain (-circular chain) covering $X$. All mappings are assumed to be continuous.

Theorem 1. A metric continuum $X$ is $k$-junctioned, where $k>0$, if and only if $X$ contains a $k$-element subset $P$ such that every subcontinuum of $X-P$ is chainable.

Proof. The "only if" part has been proved in [4, Theorem 7]. For the sake of completeness we repeat here an idea of its proof. We may assume that $X$ is not $m$-junctioned for any $m<k$. Therefore, there exist finite open covers $\mathscr{C}_{n}$ of $X$ such that mesh $\mathscr{C}_{n} \rightarrow 0$ and $\mathscr{C}_{n}$ contains exactly $k$ junction elements $C_{n}^{1}, \ldots, C_{n}^{k}$ for every $n=1,2, \ldots$. Define subsequences $\alpha_{1}, \ldots, \alpha_{k}$ of natural numbers and points $p_{1}, \ldots, p_{k}$ of $X$ by induction: $\alpha_{1}$ is a sequence $\left(n_{1}, n_{2}, \ldots\right)$ such that the sets $C_{n_{1}}^{1}, C_{n_{2}}^{1}, \ldots$ converge to a point $p_{1}$; for $1 \leq i \leq k-1, \alpha_{i+1}$ is a subsequence $\left(a_{1}, a_{2}, \ldots\right)$ of $\alpha_{i}$ such that the sets $C_{a_{1}}^{i+1}, C_{a_{2}}^{i+1}, \ldots$ converge to a point $p_{i+1}$. Put $P=\left\{p_{1}, \ldots, p_{k}\right\}$ and $\alpha_{k}=\left(a_{1}, a_{2}, \ldots\right)$. If $K$ is a subcontinuum of $X-P$, then for sufficiently large $m$ all the junction elements $C_{a_{m}}^{1}, \ldots, C_{a_{m}}^{k}$ of the cover $\mathscr{C}_{a_{m}}$ are disjoint with $K$. Therefore the family $\left\{C \in \mathscr{C}_{a_{m}}: C \cap K \neq \varnothing\right\}$ is a chain in $X$ of arbitrarily small mesh covering $K$ (it cannot be a circular chain, for $k>1$ ). It means that $K$ is chainable.

Suppose now a metric continuum $X$ contains a subset $P=\left\{p_{1}, \ldots, p_{k}\right\}$ such that every subcontinuum of $X-P$ is chainable. Let $\varepsilon>0$ be a positive number such that if $k>1$ then $\varepsilon<\min \left\{\operatorname{dist}\left(p_{i}, p_{j}\right) ; i, j=1, \ldots, k\right\}$. Consider open $\varepsilon / 2$-neighborhoods $B_{1}, \ldots, B_{k}$ of points $p_{1}, \ldots, p_{k}$, respectively. For every component $C$ of the compact set $Z=X-\bigcup_{i=1}^{k} B_{i}$ denote by $\mathscr{U}_{C}$ an $\varepsilon / 2$-chain in $X$ covering $C$. There is an open set $V_{C} \subset X$ such that $C \subset V_{C} \subset \bar{V}_{C} \subset \cup \mathscr{U}_{C}$ and bd $V_{C} \cap Z=\varnothing$. Choose a finite number of sets $V_{C_{1}}, \ldots, V_{C_{n}}$ covering $Z$. The sets $W_{i}=V_{C_{i}} \cap Z$ are closed and open in $Z$ for $i=1, \ldots, k$. Therefore the sets $T_{1}=W_{1}$ and $T_{i}=W_{i}-\left(W_{1} \cup \cdots \cup W_{i-1}\right)$, for $i=2, \ldots, n$, are closed and open in $Z$, disjoint and cover $Z$. Now, the sets $U \cap T_{i}$, where $U \in \mathscr{U}_{C}$, are open in $X$ unless

$$
U \cap T_{i} \cap \text { bd } B_{j} \neq \varnothing \quad \text { for some } j \text {. }
$$

Put, for every $j=1, \ldots, k$,

$$
G_{j}=B_{j} \cup \bigcup\left\{U: U \in \mathscr{U}_{C_{i}}, U \text { satisfies }(*) \text { and } i=1, \ldots, n\right\}
$$

and define

$$
\mathscr{G}=\left\{G_{1}, \ldots, G_{k}\right\} \cup\left\{U: U \in \mathscr{U}_{C_{i}}, U \text { does not satisfy }(*) \text { and } i=1, \ldots, n\right\} .
$$

It follows from the construction that $\mathscr{G}$ is an open $\varepsilon$-cover of $X$ of order one and the only junction elements of $\mathscr{G}$ can be $G_{1}, \ldots, G_{k}$. The proof of Theorem 1 is complete.

Theorem 2. If $f$ is a monotone mapping of a $k$-junctioned continuum $X$ onto a nondegenerate Hausdorff space $Y$, then the $Y$ is a $k$-junctioned continuum.

Proof. The result is known for $k=0[1,3]$. Suppose $k>0$ and let $P$ be a $k$ element subset of $X$ satisfying Theorem 1. In view of Theorem 1, it is enough 
to observe that every subcontinuum $K$ of $Y-f(P)$ is chainable. Indeed, $f^{-1}(K)$ is a chainable subcontinuum of $X-P$ by Theorem 1 and the mapping $f$ restricted to $f^{-1}(K)$ is monotone; so $K$ is chainable by Bing's result [1].

It is proved in [3, Theorem 3.2] that if every proper subcontinuum of a metric continuum $X$ is chainable, then so is every proper subcontinuum of an open image of $X$.

Actually, the proof gives the following statement.

Lemma [3]. If $f$ is an open mapping of a metric continuum $X$ onto a Hausdorff space and $K$ is a proper subcontinuum of $Y$ such that a component of $f^{-1}(K)$ is a chainable continuum, then the continuum $K$ is chainable.

Theorem 3. Let $f$ be an open mapping of a $k$-junctioned continuum $X$ onto a nondegenerate Hausdorff space $Y$. If $k=0$ then $Y$ is a 1-junctioned continuum. If $k>0$ then $Y$ is a $k$-junctioned continuum.

Proof. In order to prove the case $k=0$ pick a point $p \in Y$, a subcontinuum $K \subset Y-\{p\}$, and a component $C$ of $f^{-1}(K)$. The component $C$, being a proper subcontinuum of $X$, is a chainable and lemma implies $K$ is chainable. By Theorem 1 the continuum $Y$ is 1 -junctioned. The proof of the case $k>0$ is similar.

\section{REFERENCES}

1. R. H. Bing, Concerning hereditarily indecomposable continua, Pacific J. Math. 1 (1951), 43-51.

2. C. E. Burgess, Homogeneous continua which are almost chainable, Canad. J. Math. 13 (1961), 519-528.

3. E. Duda and J. Kell, Monotone and open mappings on circularly chainable continua, Proc. Internat. Conf. on Geometric Topology, PWN, Warsaw 1980, pp. 109-111.

4. E. Duda, P. Krupski, and J. T. Rogers, Jr., On locally chainable homogeneous continua (submitted).

5. W. T. Ingram, On atriodic tree-like continua with positive span, Fund. Math. 77 (1972), 99-107.

6. P. Krupski, Open images of solenoids, Topology, Proc., Leningrad 1982, Lecture Notes in Math., Springer-Verlag, Berlin and New York, vol. 1060, 1984, pp. 76-83.

7. W. Lewis, Homogeneous tree-like continua, Proc. Amer. Math. Soc. 82 (1981), 470-472.

8. I. Rosenholtz, Open maps of chainable continua, Proc. Amer. Math. Soc. 42 (1974), 258264.

Department of Mathematics and Computer Science, University of Miami, Coral GABLES, FLORIDA 33124

Mathematical Institute, University of Wroclaw, PL Grunwaldski 2/4, 50-384 WroClaw, Poland 